\title{
BOUNDS ON INVERSE DOMINATION IN SQUARES OF GRAPHS
}

\author{
M. H. Muddebihal ${ }^{1}$, Srinivasa $\mathbf{G}^{2}$ \\ ${ }^{1}$ Professor, Department of Mathematics, Gulbarga University, Karnataka, India, mhmuddebihal@yahoo.co.in \\ ${ }^{2}$ Assistant Professor, Department of Mathematics, B. N. M. I. T, Karnataka, India, gsgraphtheory@yahoo.com
}

\begin{abstract}
Let $D$ be a minimum dominating set of a square graph $G^{2}$. If $V\left(G^{2}\right)-D$ contains another dominating set $D^{\prime}$ of $G^{2}$, then $D^{\prime}$ is called an inverse dominating set with respect to $D$. The minimum cardinality of vertices in such a set is called an inverse domination number of $G^{2}$ and is denoted by $\gamma^{-1}\left(G^{2}\right)$. In this paper, many bounds on $\gamma^{-1}\left(G^{2}\right)$ were obtained in terms of elements of $G$. Also its relationship with other domination parameters was obtained.
\end{abstract}

Key words: Square graph, dominating set, inverse dominating set, Inverse domination number.

Subject Classification Number: AMS-05C69, 05C70

\section{INTRODUCTION}

In this paper, we follow the notations of [1]. We consider only finite undirected graphs without loops or multiple edges. In general, we use $\langle X\rangle$ to denote the subgraph induced by the set of vertices $X$ and $N(v)$ and $N[v]$ denote the open and closed neighborhoods of a vertex $v$.

The minimum (maximum) degree among the vertices of $G$ is denoted by $\delta(G)(\Delta(G))$. A vertex of degree one is called an end vertex. The term $\alpha_{0}(G)\left(\alpha_{1}(G)\right)$ denotes the minimum number of vertices(edges) cover of $G$. Further, $\beta_{0}(G)\left(\beta_{1}(G)\right)$ represents the vertex(edge) independence number of $G$.

A vertex with degree one is called an end vertex. The distance between two vertices $u$ and $v$ is the length of the shortest uv-path in $G$. The maximum distance between any two vertices in $G$ is called the diameter of $G$ and is denoted by $\operatorname{diam}(G)$.

The square of a graph $G$ denoted by $G^{2}$, has the same vertices as in $G$ and the two vertices $u$ and $v$ are joined in $G^{2}$ if and only if they are joined in $G$ by a path of length one or two. The concept of squares of graphs was introduced in [2].
A set $S \subseteq V(G)$ is said to be a dominating set of $G$, if every vertex in $(V-S)$ is adjacent to some vertex in $S$. The minimum cardinality of vertices in such a set is called the domination number of $G$ and is denoted by $\gamma(G)$. Further, if the subgraph $\langle S\rangle$ is independent, then $S$ is called an independent dominating set of $G$. The independent omination number of $G$, denoted by $\gamma_{i}(G)$ is the minimum cardinality of an independent dominating set of $G$.

A dominating set $S$ of $G$ is said to be a connected dominating set, if the subgraph $\langle S\rangle$ is connected in $G$. The minimum cardinality of vertices in such a set is called the connected domination number of $G$ and is denoted by $\gamma_{c}(G)$.

A dominating set $S$ is called total dominating set, if for every vertex $v \in V$, there exists a vertex $u \in S, u \notin v$ such that $u$ is adjacent to $v$. The total domination number of $G$, denoted by $\gamma_{t}(G)$ is the minimum cardinality of total dominating set of $G$.

Further, a dominating set $S$ is called an end dominating set of $G$, if $S$ contains all the end vertices in $G$. The minimum cardinality of vertices in such a set is called the end domination number of $G$ and is denoted by $\gamma_{e}(G)$. 
Domination related parameters are now well studied in graph theory (see [3] and [9]).

Let $S$ be a minimum dominating set of $G$, if the compliment $V-S$ of $S$ contains a dominating set $S^{\prime}$, then $S^{\prime}$ is called an inverse dominating set of $G$ with respect to $S$. The minimum cardinality of vertices in such a set is called an inverse domination number of $G$ and is denoted by $\gamma^{-1}(G)$. Inverse domination was introduced by V. R. Kulli and S. C. Sigarkanti [10].

A set $D \subseteq V\left(G^{2}\right)$ is said to be a dominating set of $G^{2}$, if every vertex not in $D$ is adjacent to a vertex in $D$. The minimum cardinality of vertices in such a set is called the domination number of $G^{2}$ and is denoted by $\gamma\left(G^{2}\right)$ (see [4]).

A set $D$ of $G^{2}$ is said to be connected dominating set of $G^{2}$ if every vertex not in $D$ is adjacent to at least one vertex in $D$ and the set induced by $D$ is connected. The minimum cardinality of a connected dominating set of $G^{2}$ is called connected domination number of $G^{2}$ (see [5]).

A dominating set $D$ of $G^{2}$ is said to be total dominating set, if for every vertex $v \in V\left(G^{2}\right)$, there exists a vertex $u \in D, u \neq$ $v$, such that $u$ is adjacent to $v$. The total domination number of $G^{2}$ denoted by $\gamma_{t}\left(G^{2}\right)$ is the minimum cardinality of a total dominating set of $G^{2}$ (see [6]).

A dominating set $D$ of $G^{2}$ is said to be restrained dominating set, if for every vertex not in $D$ is adjacent to a vertex in $D$ and to a vertex in $(V-D)$. The restrained domination number of $G^{2}$, denoted by $\gamma_{r e}\left(G^{2}\right)$ is the minimum cardinality of a restrained dominating set of $G^{2}$. Further, a dominating set $D$ of $G^{2}$ is said to be double dominating set, if for every vertex $v \in V\left(G^{2}\right)$ is dominated by at least two vertices of $D$. The double domination number of $G^{2}$, denoted by $\gamma_{d}\left(G^{2}\right)$ is the minimum cardinality of a double dominating set of $G^{2}$ (see [7] and [8]).
Analogously, let $D$ be a minimum dominating set of a square graph $G^{2}$. If $V\left(G^{2}\right)-D$ contains another dominating set $D^{\prime}$ of $G^{2}$, then $D^{\prime}$ is called an inverse dominating set with respect to $D$. The minimum cardinality of vertices in such a set is called an inverse domination number of $G^{2}$ and is denoted by $\gamma^{-1}\left(G^{2}\right)$. In this paper, many bounds on $\gamma^{-1}\left(G^{2}\right)$ were obtained in terms of elements of $G$. Also its relationship with other domination parameters was obtained.

\section{RESULTS}

Theorem 2.1: For any connected graph $G, \gamma^{-1}\left(G^{2}\right)+$ $\gamma_{t}\left(G^{2}\right) \leq \delta(G)+\gamma_{t}(G)$.

Proof: Let $S=\left\{v_{1}, v_{2}, \ldots, v_{m}\right\} \subseteq V(G)$ be the minimum set of vertices which covers all the vertices in G. Suppose the subgraph $\langle S\rangle$ has no isolated vertices, then $S$ itself is a $\gamma_{t}$ set of $G$. Otherwise, let $B=\left\{v_{1}, v_{2}, \ldots, v_{n}\right\} \subseteq S$ be the set of vertices with $\operatorname{deg}\left(v_{i}\right)=D, 1 \leq i \leq n$. Now make $\operatorname{deg}\left(v_{i}\right)=$ 1 by adding vertices $\left\{u_{i}\right\} \subseteq V(G)-S$ and $N\left(v_{i}\right) \in\left\{u_{i}\right\}$. Clearly, $S_{1}=S \cup B \cup\left\{u_{i}\right\}$ forms a minimal total dominating set of $G$. Since $V(G)=V\left(G^{2}\right)$, let $D_{t}=\left\{v_{1}, v_{2}, \ldots, v_{k}\right\} \subseteq S_{1}$ be the minimal $\gamma_{t}$-set of $G^{2}$. Suppose $D \subseteq S$ is a minimal dominating set of $G^{2}$. Then there exists a vertex set $D^{\prime}=$ $\left\{v_{1}, v_{2}, \ldots, v_{k}\right\} \subseteq V\left(G^{2}\right)-D$ which covers all the vertices in $G^{2}$. Clearly, $D^{\prime}$ forms a minimal inverse dominating set $G^{2}$. Since for any graph $G$, there exists atleast one vertex $v \in V(G)$, such that $\operatorname{deg}(v)=\delta(G)$, it follows that, $\left|D^{\prime}\right| \cup$ $\left|D_{t}\right| \leq\left|S_{1}\right|+\operatorname{deg}(v) . \quad$ Therefore, $\quad \gamma^{-1}\left(G^{2}\right)+\gamma_{t}\left(G^{2}\right) \leq$ $\gamma_{t}(G)+\delta(G)$.

Theorem 2.2: Let $D$ and $D^{\prime}$ be, $\gamma$-set and $\gamma^{-1}$-set of $G^{2}$ respectively. If $\gamma\left(G^{2}\right)=\gamma^{-1}\left(G^{2}\right)$, then each vertex in $D$ is of maximum degree.

Proof: For $p=2$, the result is obvious. Let $p \geq 3$. Since, $V(G)=V\left(G^{2}\right)$ such that $G^{2}$ doesnot contain any end vertex, let $D=\left\{v_{1}, v_{2}, \ldots, v_{n}\right\}$ be a dominating set of $G^{2}$. If there exists a vertex $v \in D$ such that $v$ is adjacent to some vertices in $V\left(G^{2}\right)-D$. Then every vertex $w \in D-\{v\}$ is an end vertex in $\langle D\rangle$. Further, if $w$ is adjacent to a vertex 
$u \in V\left(G^{2}\right)-D$. Then $D^{\prime}=D-\{v, w\} \cup\{u\}$ is a dominating set of $G^{2}$, a contradiction. Hence each vertex in $D$ is of maximum degree in $G^{2}$.

Theorem 2.3: For any connected graph $G, \gamma^{-1}\left(G^{2}\right)=1$ if and only if $G^{2}$ has at least two vertices of degree $(p-1)$.

Proof: To prove this result, we consider the following two cases.

Case 1. Suppose $G^{2}$ has exactly one vertex $v$ of $\operatorname{deg}(v)=$ $p-1$. Then in this case $D=\{v\}$ is a $\gamma$-set of $G^{2}$. Clearly, $V-D=V-\{v\}$. Further, if $D_{1}=\{u\} \in N(v)$ in $V\left(G^{2}\right)-D$, $\operatorname{deg}(u) \leq p-2$ in $G^{2}$. Then there exists at least one vertex $w \notin N(u)$ in $G^{2}$ such that $D^{\prime}=D_{1} \cup\{w\}$ forms an inverse dominating set of $G^{2}$, a contradiction.

Case 2. Suppose $G^{2}$ has at least two vertices $u$ and $v$ of $\operatorname{deg}(u)=p-1=\operatorname{deg}(v)$ such that $u$ and $v$ are not adjacent. Then $D=\{u\}$ dominates $G^{2}$ since $\operatorname{deg}(u)=p-$ 1 and $V\left(G^{2}\right)-D=V\left(G^{2}\right)-\{u\}$. Further, since $u$ and $v$ are not adjacent, $D^{\prime}=\{v\} \cup V^{\prime}$ where $V^{\prime} \subseteq V\left(G^{2}\right)-D$ forms a $\gamma^{-1}$-set of $G^{2}$, a contradiction.

Conversely, suppose $\operatorname{deg}(u)=p-1=\operatorname{deg}(v)$, such that $u$ and $v$ are adjacent to all the vertices in $G^{2} . D^{\prime}=\{v\} \in N(u)$, where $\{v\} \subseteq V\left(G^{2}\right)-D$ and vice-versa. In any case, we obtain $\left|D^{\prime}\right|=1$. Therefore $\gamma^{-1}\left(G^{2}\right)=1$.

Proposition 2.1: $\quad \gamma^{-1}\left(K_{p}^{2}\right)=\gamma^{-1}\left(W_{p}^{2}\right)=\gamma^{-1}\left(K_{1, p}^{2}\right)=$ $\gamma^{-1}\left(K_{p_{1}, p_{2}}^{2}\right)=1$

Theorem 2.4: For any connected $(p, q)$-graph $G$ without isolates, then $\gamma\left(G^{2}\right)+\gamma^{-1}\left(G^{2}\right) \leq p$.

Proof : Suppose $D=\left\{v_{1}, v_{2}, \ldots, v_{n}\right\} \subseteq V\left(G^{2}\right)$ be the $\gamma$-set of $G^{2}$, the $D^{\prime}=\left\{v_{1}, v_{2}, \ldots, v_{n}\right\} \subseteq V\left(G^{2}\right)-D$ forms a minimal inverse dominating set of $G^{2}$. Since $|D| \leq\lceil p / 4\rceil$ and $\left|D^{\prime}\right| \leq\lceil p / 3]$, it follows that, $|D| \cup\left|D^{\prime}\right| \leq p$. Therefore, $\gamma\left(G^{2}\right)+\gamma^{-1}\left(G^{2}\right) \leq p$.
Suppose $V-D$ is not independent, then there exists at least one vertex $u \in D^{\prime}$ such that $N(u) \subseteq V-D$. Clearly, $\left|D^{\prime}\right|=$ $|\{v-D\}-\{u\}|$ and hence, $|D| \cup\left|D^{\prime}\right| \leq p$, a contradiction.

Conversely, if $V-D$ is independent. Then in this case, $\left|D^{\prime}\right|=|V-D|$ in $G^{2}$. Clearly, it follows that $|D| \cup\left|D^{\prime}\right|=p$. Hence, $\gamma\left(G^{2}\right)+\gamma^{-1}\left(G^{2}\right)=p$.

Theorem 2.5: For any connected $(p, q)$-graph $G$ with $p \geq 3$ vertices, $\gamma^{-1}\left(G^{2}\right)+\gamma_{i}(G) \leq p-1$.

Proof: For $p \leq 2, \gamma^{-1}\left(G^{2}\right)+\gamma_{i}(G) \not p-1$. Consider $p \geq 3$, let $F=\left\{v_{1}, v_{2}, \ldots, v_{m}\right\}$ be the minimum set of vertices such that for every two vertices $u, v \in F, N(u) \cap N(v) \in$ $V(G)-F$. Suppose there exists a vertex $S=\left\{v_{1}, v_{2}, \ldots, v_{k}\right\} \subseteq F$ which covers all the vertices in $G$ and if the subgraph $\langle S\rangle$ is totally disconnected. Then $\mathrm{S}$ forms the minimal independent dominating set of $G$. Now in $G^{2}$, since $V(G)=V\left(G^{2}\right)$ and distance between two vertices is at most two in $G^{2}$, there exists a vertex set $D=$ $\left\{v_{1}, v_{2}, \ldots, v_{j}\right\} \subseteq S$, which forms minimal $\gamma$-set of $G^{2}$. Then the complementary set $V\left(G^{2}\right)-D$ contains another set $D^{\prime}$ such that $N\left(D^{\prime}\right)=V\left(G^{2}\right)$. Clearly, $D^{\prime}$ forms an inverse dominating set of $G^{2}$ and it follows that $\left|D^{\prime}\right| \cup|S| \leq p-1$. Therefore, $\gamma^{-1}\left(G^{2}\right)+\gamma_{i}(G) \leq p-1$.

Theorem 2.6: If every non end vertex of a tree is adjacent to at least one end vertex, then, $\gamma^{-1}\left(T^{2}\right) \leq\left\lceil\frac{p-m}{2}\right\rceil+1$.

Proof: Let $F=\left\{v_{1}, v_{2}, \ldots, v_{m}\right\}$ be the set of all end vertices in $T$ such that vertex $|F|=m$ and $F_{1} \in N(F)$. Suppose, vertex $D \subseteq F_{1}$ is a $\gamma$-set of $G^{2}$. Then $D^{\prime} \subseteq V\left(G^{2}\right)-D-$ $F$ forms a minimal inverse dominating set of $G^{2}$. Since every tree $T$ contains at least one non end vertex, it follows that $\left|D^{\prime}\right| \leq\left\lceil\frac{p-m}{2}\right\rceil+1$. Therefore, $\gamma^{-1}\left(T^{2}\right) \leq\left\lceil\frac{p-m}{2}\right\rceil+1$.

Theorem 2.7: For any connected $(p, q)$-graph $G, \gamma^{-1}\left(G^{2}\right)+$ $\gamma_{c}(G) \leq p+\gamma(G)$. 
Proof: For $P \leq 5$, the result follows immediately. Let $P \geq 6$, suppose $D=\left\{v_{1}, v_{2}, \ldots, v_{n}\right\}, \operatorname{deg}\left(v_{i}\right) \geq 2,1 \leq i \leq n$ be a minimal dominating set of $G$. Now we construct a connected dominating set $D_{c}$ from $D$ by adding in every step at most two components of $D$ forms a connected component in $D$. Thus we get a connected dominating set $D_{c}$ after at most $D-1$ steps. Now in $G^{2}, V(G)=V\left(G^{2}\right)$. Suppose $D_{1} \subseteq D$ be minimal $\gamma$-set of $G^{2}$. Then there exists a vertex set $D^{\prime}=$ $\left\{v_{1}, v_{2}, \ldots, v_{k}\right\} \subseteq V\left(G^{2}\right)-D_{1}$, such that $\operatorname{dist}(u, v) \geq 2$, for all $u, v \in D^{\prime}$, which covers all the vertices in $G^{2}$. Clearly, $D^{\prime}$ forms a minimal inverse dominating set of $G^{2}$. Hence it follows that $\left|D^{\prime}\right| \cup\left|D_{c}\right| \leq p \cup|D|$. Therefore, $\gamma^{-1}\left(G^{2}\right)+$ $\gamma_{c}(G) \leq p+\gamma(G)$.

Theorem 2.8: For any connected $(p, q)$-graph $G$, $\gamma^{-1}\left(G^{2}\right)+\gamma_{c}\left(G^{2}\right) \leq \gamma\left(G^{2}\right)+\beta_{0}(G)$. Equality holds for $K_{p}$.

Proof: Let $B=\left\{v_{1}, v_{2}, \ldots, v_{k}\right\}$ be the maximum set of vertices such that $\langle B\rangle$ is totally disconnected and $|B|=$ $\beta 0 G$. Now in $G 2$, let $D=v 1, v 2, \ldots, v n \subseteq V G 2$ be the minimum set of vertices such that $N(D)=V\left(G^{2}\right)$. Clearly, $D$ forms a minimal $\gamma$-set of $G^{2}$. Suppose the subgraph $\langle D\rangle$ is connected, then $D$ itself is a connected dominating set of $G^{2}$. Otherwise, construct the connected dominating set $D_{c}$ from $D$ by adding at most two vertices $u, v \notin D$ between the vertices of $D$ such that $N\left(D_{c}\right)=V\left(G^{2}\right)$ and $\left\langle D_{c}\right\rangle$ is connected. Further, there exists a vertex set $D^{\prime}=\left\{u_{1}, u_{2}, \ldots, u_{n}\right\} \subseteq$ $V\left(G^{2}\right)-D$ which covers all the vertices in $G^{2}$. Clearly, $D^{\prime}$ forms an inverse dominating set of $G^{2}$ with respect to $\gamma$-set of $G^{2}$. Since $\operatorname{diam}(u, v) \geq 2, \forall u, v \in V\left(G^{2}\right)$, it follows that $\left|D^{\prime}\right| \cup\left|D_{c}\right| \leq|D| \cup|B|$. Therefore, $\quad \gamma^{-1}\left(G^{2}\right)+\gamma_{c}\left(G^{2}\right) \leq$ $\gamma\left(G^{2}\right)+\beta_{0}(G)$.

Suppose $G \cong K_{p}$. Then in this case, $|B|=|D|=\left|D_{c}\right|=$ $\left|D^{\prime}\right|=1$. Therefore, $\gamma^{-1}\left(G^{2}\right)+\gamma_{c}\left(G^{2}\right)=\gamma\left(G^{2}\right)+\beta_{0}(G)$.

Theorem 2.9: For any connected $(p, q)$-graph $G, \gamma^{-1}\left(G^{2}\right) \leq$ $\left\lceil\frac{p-\gamma_{e}(G)}{2}\right\rceil+3$.
Proof: Let $F=\left\{v_{1}, v_{2}, \ldots, v_{n}\right\}$ be the set of all end vertices in $G$ and let $v_{1} \notin N[F]$ in $G$. Suppose $H \subseteq V_{1}$ is a dominating set of the subgraph $\left\langle V_{1}\right\rangle$. Then $F \cup H$ forms a minimal end dominating set of $G$. In $G^{2}, V(G)=V\left(G^{2}\right)$. Suppose $\operatorname{diam}(G) \leq 3$, then $D^{\prime}=\{u, v\}$ is a minimal $\gamma^{-1}$-set of $G^{2}$ with respect to $\gamma$-set $D$ of $G^{2}$, and the result follows immediately. Suppose $\operatorname{diam}(G) \geq 4$, then $D^{\prime}=$ $\left\{u_{1}, u_{2}, \ldots, u_{k}\right\} \subseteq V\left(G^{2}\right)-D-F$ is a minimal inverse dominating set of $G^{2}$ with respect to the dominating set $D$ of $G^{2}$. Clearly, it follows that $\left|D^{\prime}\right| \leq\left\lceil\frac{p-\{F \cup H\}}{2}\right\rceil+3$. Therefore, $\gamma^{-1}\left(G^{2}\right) \leq\left\lceil\frac{p-\gamma_{e}(G)}{2}\right\rceil+3$

Theorem 2.10: For any connected $(p, q)$-graph $G$, $\gamma^{-1}\left(G^{2}\right)+\gamma_{r e}\left(G^{2}\right) \leq p-\alpha_{0}(G)+1$. Equality holds for $K_{p}, P_{6}$.

Proof: Let $C=\left\{v_{1}, v_{2}, \ldots, v_{i}\right\}$ be the minimal set of vertices which covers all the edges in $G$ such that $|C|=\alpha_{0}(G)$. Now in $G^{2}$,obtain $H$, the induced subgraph of $G^{2}$, i.e., $H=\left\langle G^{2}>\right.$ such that $H$ contains at least one end vertex $v \in H$. Let $F=$ $\left\{v_{1}, v_{2}, \ldots, v_{k}\right\}$ be the set of all such end vertices in $G^{2}$. Suppose $V^{\prime}=V\left(G^{2}\right)-F$ and $\quad I \subseteq V^{\prime}$, such that $\operatorname{diam}(u, v) \geq 2$, for all $u \in I$ and $v \in F$. Then $D=F \cup I^{\prime}$, where $I^{\prime} \subseteq I$, covers all the vertices in $G^{2}$ such that every vertex not in $D$ is adjacent to a vertex in $D$ and to a vertex in $V\left(G^{2}\right)-D$. Clearly, $D$ forms a minimal restrained dominating set of $G^{2}$. Let $D^{\prime}=\left\{w_{1}, w_{2}, \ldots, w_{n}\right\} \subseteq V\left(G^{2}\right)$ forms a minimal $\gamma^{-1}$-set of $G^{2}$ with respect to dominating set of $G^{2}$. Clearly, it follows that $\left|D^{\prime}\right| \cup|D| \leq p-|C|+1$. Therefore, $\gamma^{-1}\left(G^{2}\right)+\gamma_{r e}\left(G^{2}\right) \leq p-\alpha_{0}(G)+1$.

For equality, suppose $G \cong K_{p}$. Then in this case, $|D|=1=$ $\left|D^{\prime}\right|$ and $|C|=p-1$. Therefore, $\left|D^{\prime}\right| \cup|D|=p-|C|+1$ and hence $\gamma^{-1}\left(G^{2}\right)+\gamma_{r e}\left(G^{2}\right)=p-\alpha_{0}(G)+1$.

Suppose $G \cong P_{6}$. Then in this case, $|D|=2=\left|D^{\prime}\right|$ and $|C|=\frac{p}{2}$. Clearly, it follows that, $\gamma^{-1}\left(G^{2}\right)+\gamma_{r e}\left(G^{2}\right)=p-$ $\alpha_{0}(G)+1$. 
Theorem 2.11: For any connected $(p, q)$-graph $G, \gamma^{-1}\left(G^{2}\right)+\gamma_{d}\left(G^{2}\right) \leq 2 p-q+1$. Equality holds for $K_{p, p} \geq 4$.

Proof: Let $I=\left\{v_{1}, v_{2}, \ldots, v_{n}\right\} \subseteq V\left(G^{2}\right)$ and let $I^{\prime} \in N(I)$, for every vertex $v \in I$ in $G^{2}$. Now we form the vertex set $X \subseteq V\left(G^{2}\right)$ by random and independent choice of the vertices of $I$. Define $X_{0}=\{I \in X:|N(I) \cap X|=\emptyset\}, Y_{0}=$ $\{I \notin X:|N(I) \cap X|=\emptyset\}$ and $\quad Y_{1}=\{I \notin X:|N(I) \cap X|=I\}$. Further, if $X_{0}^{\prime}=\bigcup_{I \in X_{0}}\left\{I^{\prime}\right\}$ and $Y_{0}^{\prime}=\bigcup_{I \in Y_{0}}\left\{I^{\prime}\right\}$. Then $\left|X_{0}^{\prime}\right| \leq\left|X_{0}\right|$ and $\left|Y_{0}^{\prime}\right| \leq\left|Y_{0}\right|$. Clearly, the set $D_{d}=X \cup$ $X O^{\prime} \cup Y O \cup Y O^{\prime} \cup Y 1$ forms a $\gamma d$-set of $G 2$. Let $D^{\prime}=u 1, u 2, \ldots, u k$ be the minimal $\gamma^{-1}$-set of $G^{2}$ with respect to dominating set $D$ of $G^{2}$. It follows that $\left|D^{\prime}\right| \cup\left|D_{d}\right| \leq 2 p-q+1$ and hence $\gamma^{-1}\left(G^{2}\right)+\gamma_{d}\left(G^{2}\right) \leq 2 p-q+1$.

For equality, suppose $G \cong K_{p}$, with $p \geq 4$ vertices. Then in this case, $\left|D_{d}\right|=2=2(1)=2\left|D^{\prime}\right|$ and $\mathrm{q}=\frac{\mathrm{p}(\mathrm{p}-1)}{2}$ for $K_{p}$. Clearly, $\quad\left|D^{\prime}\right| \cup\left|D_{d}\right|=2 p-q+1$ Hence $\gamma^{-1}\left(G^{2}\right)+\gamma_{d}\left(G^{2}\right)=2 p-q+1$.

Theorem 2.12: For any connected $(p, q)$ - graph $G$, $\gamma^{-1}\left(G^{2}\right)+\operatorname{diam}(G) \leq p+\gamma(G)-1$.

Proof: Suppose $G \cong K_{2}$, then obviously, $\gamma^{-1}\left(G^{2}\right)+$ $\operatorname{diam}(G)=p+\gamma(G)-1$. Let $V(G)$ contains atleast two vertices $u$ and $v$ such that $\operatorname{dist}(u, v)$ forms a diametral path in $G$. Clearly, $\operatorname{dist}(u, v)=\operatorname{diam}(G)$. Let $F_{1}=\left\{v_{1}, v_{2}, \ldots, v_{k}\right\} \subseteq V(G)$ be the set of vertices which are adjacent to all end vertices in $G$. Suppose $J=\left\{u_{1}, u_{2}, \ldots, u_{n}\right\}$ be the set of vertices such that $\operatorname{dist}\left(v_{i}, u_{j}\right) \geq 2$, for all $1 \leq i \leq k, 1 \leq j \leq n$. Then $F_{1} \cup J^{\prime}$ where $J^{\prime} \subseteq J$ forms a minimal $\gamma$-set of $G$. Now in $G^{2}$, suppose $D=\left\{v_{1}, v_{2}, \ldots, v_{m}\right\} \subseteq F_{1} \cup J^{\prime}$ be the minimal $\gamma$-set of $G^{2}$. Then the complementary set $\mathrm{V}\left(\mathrm{G}^{2}\right)-\mathrm{D}$ contains the vertex set $D^{\prime} \subseteq \mathrm{V}\left(\mathrm{G}^{2}\right)-\mathrm{D}$, which covers all the vertices in $G^{2}$. Clearly, $D^{\prime}$ forms a minimal inverse dominating set of $G^{2}$ and it follows that $\left|D^{\prime}\right|+\operatorname{diam}(G) \leq p+\left|F_{1} \cup J^{\prime}\right|-1$. Hence $\gamma^{-1}\left(G^{2}\right)+\operatorname{diam}(G) \leq p+\gamma(G)-1$.

\section{REFERENCES}

[1]. F. Harary, Graph Theory, Adison-Wesley, Reading, Mass., 1972.

[2]. F. Harary and I. C. Ross, The Square of a Tree, Bell System Tech. J. 39, 641-647, 1960.

[3]. M. A. Henning, Distance Domination in Graphs, in: T. W. Haynes, S. T. Hedetniemi and P. J. Slater, editors. Domination in Graphs: Advanced Topics, chapter 12, Marcel Dekker, Inc., New York, 1998.

[4]. M. H. Muddebihal, G. Srinivasa and A. R. Sedamkar, Domination in Squares of Graphs, Ultra Scientist, 23(3)A, 795-800, 2011.

[5]. M. H. Muddebihal and G. Srinivasa, Bounds on Connected Domination in Squares of Graphs, International Journal of Science and Technology, 1(4), 170-176, April 2012.

[6]. M. H. Muddebihal and G. Srinivasa, Bounds on Total Domination in Squares of Graphs, International Journal of Advanced Computer and Mathematical Sciences, 4(1), 67-74, 2013.

[7]. M. H. Muddebihal and G. Srinivasa, Bounds on Restrained Domination in Squares of Graphs, International Journal of Mathematical Sciences, 33(2), 1173-1178, August 2013.

[8]. M. H. Muddebihal and G. Srinivasa, Bounds on Double Domination in Squares of Graphs, International Journal of Research in Engineering and Technology, 2(9), 454458, September 2013.

[9]. T. W. Haynes, S. T. Hedetniemi and P. J. Slater, Fundamentals of Domination in Graphs, Marcel Dekker, Inc., New York, 1998.

[10]. V. R. Kulli and S. C. Sigarkanti, Inverse Domination in Graphs. Nat. Acad. Sci. Lett., 14, 473-475, 1991. 